\title{
Plasma Treatment and Polymerization of Textile Reinforcing Materials
}

\author{
Hudec Ivan ${ }^{1, *}$, Jaššo MichaL ${ }^{1}, \mathrm{Krump} \mathrm{HeNRICH}^{1}, \check{C ̆ e r n a ́ k ~ M i R K O}^{2}$ and \\ Šuriová VIERA ${ }^{3}$ \\ ${ }^{1}$ Department of Plastics and Rubber, Faculty of Chemical and Food Technology, Slovak University of Technology, \\ Radlinského 9, 81237 Bratislava, Slovakia \\ ${ }^{2}$ Faculty of Mathematics, Physics and Informatics Commenius University, Bratislava, Slovakia \\ ${ }^{3}$ Rubber Research Institute Matador, a.s. Púchov, Slovak Republic \\ * Corresponding author: ivan.hudec@stuba.sk \\ Received July 30, 2005; Revised February 18, 2006; Accepted February 21, 2006 \\ (C) 2006 The Society of Rubber Industry, Japan
}

\begin{abstract}
In this paper different types of surface modification of polyester cords by low-temperature plasma at atmospheric pressure was studied.

The first type cords were activated by pulse surface positive corona discharge generated in a plasma reactor or by coplanar dielectric surface barier discharge (DCSBD) in nitrogen or ambient air plasma at atmospheric pressure. The values of the static and dynamic adhesion of untreated cords and the plasma treated cords demonstrated possitive influence of plasma surface treatment on the adhesion of cords to rubber. The mechanical properties were not significantly affected by plasma treatment.

The second type of plasma treatment involved the modification of cords by plasma polymerization in mixture of nitrogene with butadiene. The plasma layer homogenously coated the cords surface. The results shove, that values of static and dynamic adhesion for plasma polymerisation of treated cords are comparable with the standard chemical treatment based on resorcinol-formaldehyde latex (RFL).

From the study of the surface properties of the plasma treated cords by SEM, AFM and XPS is evident that both chemical interactions and morphological changes of the surface cord fibres are responsible for the improved adhesion between treated reinforcing materials and rubber blend.
\end{abstract}

Keywords Adhesion strength, Atmospheric plasma treatment, Polyester cord, Plasma polymerization.

\section{Introduction}

The high-performance advanced of polyester (PET) cords are superior to the conventional rubber reinforcing materials, because they have a very high strength, dimensional stability, toughness, fatigue resistance as well as lower specific weight. All those mentioned advantages are very important for the tyre industry. However, in dynamic conditions, stresses acting on the rubber matrix are transmitting to the fibre across the interface. Consequently the sufficient interfacial adhesion strength between rubber matrix and fibre surface is necessary. The key factor is the interactions between functional groups at the fibre surface and functional groups of the rubber matrix. The relatively inert chemical structure of PET shows many of desirable properties contributing adversely to their ability to bond to rubber compare with rayon and nylon ${ }^{1)}$.

Plasma techniques have been utilized in materials science since 1960s. For instance, plasma processes have been utilized to improve the surface properties of fibres in many applications. The fibres, which can be modified by plasma, include almost all kinds of fibres, such as metallic, glass, carbon and polymeric fibres. Some investigations were done to improve wettability, shrinking resistance, twisting of fibres in textile, dyeing, and printing industries. However, the focus has been on fibre-reinforced composites, especially the increasing of the interface adhesion between fibres and matrix ${ }^{2}$.

When polymeric materials are exposed to plasma, radicals are created in the polymeric chains. As results of inelastic collisions of electrons in plasma with a polymer surface and excitation of the polymer molecules, these radicals can initiate reactions when they are in contact with monomers in a liquid or gaseous phase. As a result, grafting or polymerized layer is formed on the surface of polymeric material.

Some authors tried to use low temperature plasma at 
atmospheric pressure as a method for the achievement the better adhesion strength between fibres and a polymeric matrix $^{3-5)}$

\section{Experimental}

\section{Materials}

Chemically untreated PET cords HMLS (High Modulus Low Shrinkage) was supplied by SH Senica Slovkord, Slovakia $\left(\mathrm{T}_{\mathrm{m}}=255^{\circ} \mathrm{C}, 144 \mathrm{Tex}\right.$, spin-finish 380/370). These cords are used for the carcass plies of radial tyres.

The conventional rubber blend based on SBR used as the rubber blend was supplied by Matador a.s. Púchov, Slovakia (Mooney viscosity $=41^{\circ} \mathrm{ML}$, modulus [100\%1.874 MPa, 200\%-4.814 MPa, 300\%-8.606 MPa]).

\section{Modification of PET cords by plasma}

The modification of PET cords was carried out in a laboratory reactor at atmospheric pressure. It consisted of an electrode system enclosed in a glass chamber with a gas flow. The grounded stainless-steel tubular anode had a $1 \mathrm{~mm}$ inner diameter. The cathode was a $15 \mathrm{~mm}$ diameter hemi-spherically capped brass rod with a $2 \mathrm{~mm}$ diameter

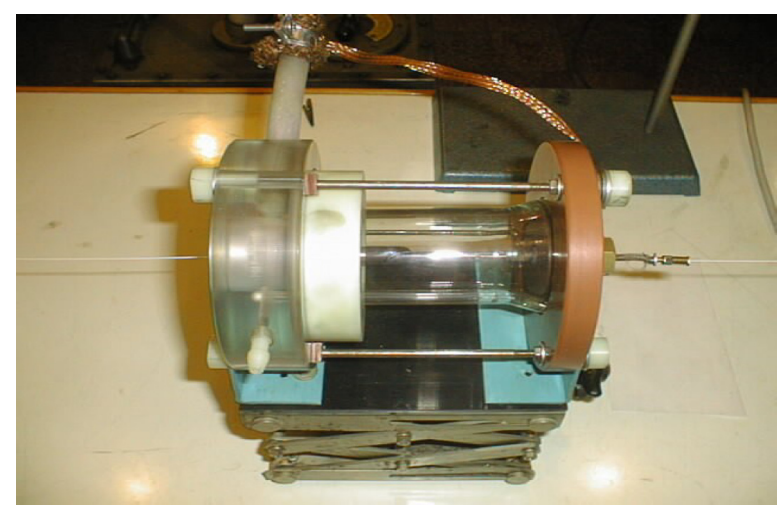

Figure 1. Atmospheric plasma reactor. hole on the axis. The treated fibre moved in the axis of the electrode system with a speed from 1.5 to $60 \mathrm{~m}$ per minute. The distance between the electrodes was adjusted to 15 $\mathrm{mm}$. Cathode was connected with a tyratron source of pulsed high voltage (HV). The HV pulse rate was maintained at $100 \mathrm{~Hz}$ and the peak voltage was approximately $25 \mathrm{kV}$. Nitrogen of a technical purity was introduced into the chamber with a flow rate on the order of $1 \mathrm{~cm}^{3} \cdot \mathrm{s}^{-1}$.

In the case of plasma polymerization in mixture of nitrogen with butadiene, the cathode was connected with a double spinning spark gab source of pulsed high voltage. The HV pulse rate was maintained at $20 \mathrm{~Hz}$ and the voltage of source was approximately $50 \mathrm{kV}$. Nitrogen of a technical purity was introduced into the chamber with a flow rate on the order of 3 litter per minute and flow rate of butadiene was $0.1,0.25$ and 0.5 litter per minute. The plasma layer homogenously coating the cord surface was generated by a pulse surface positive corona discharge at atmospheric gas pressure (Figure 1). Other parameters of a reactor remain the same.

Plasma treatment and polymerization by coplanar dielectric surface barrier discharge (DCSBD) was carried out by two parallel electrodes systems. A sinusoidal high frequency high-voltage was applied between the electrodes. A schematic diagram of the discharge electrode system is shown in Figure 2. Discharge electrode arrangement and energy level allow generating visually almost uniform plasmas in nitrogen and in the ambient air. The atmospheric pressure with the gas flow of 3 litters per minute was used.

\section{Adhesion strength testing}

The PET fibres were washed 5 minutes in acetone before plasma modification due to removing of preadhesion surface treatment from the PET surface ${ }^{6)}$. Then, the plasma treatment was applied. The samples received
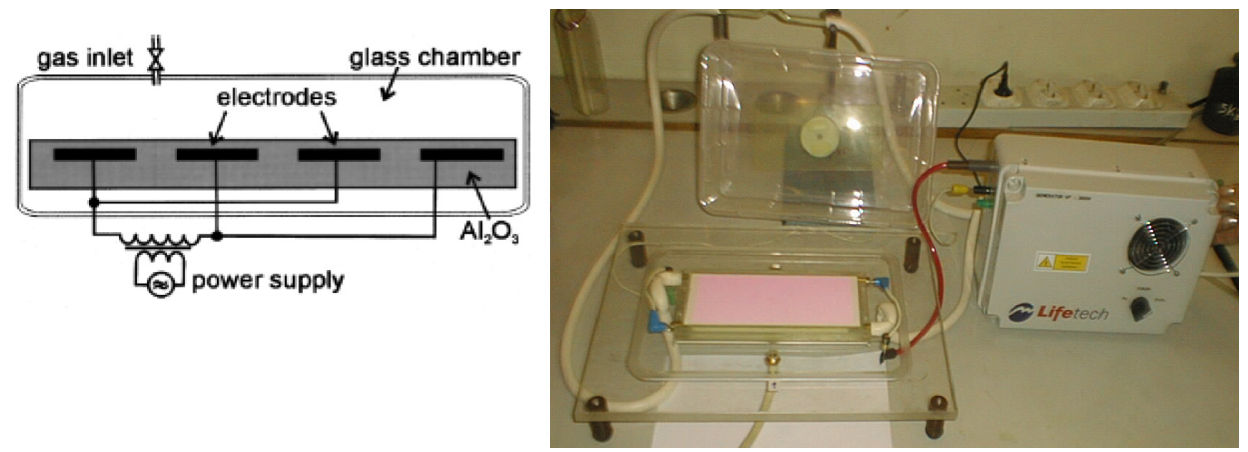

Figure 2. Schematic of the discharge electrode system and configuration of coplanar dielectric surface barrier discharge. 
from plasma treatment and plasma polymerization were prepared by pressing the cords thread between two slides from rubber blend, followed by subsequent vulcanization. Adhesion strength to rubber blend was studied by the standard Henley test according to STN 6201464. The centre of the sample was subjected to cyclic flexural stress (frequency $=7.5 \mathrm{~Hz}$ ) for 12 hours in a chamber at $80 \pm 1.5^{\circ} \mathrm{C}$. Then the sample was cut in three parts. The adhesion strength between the cord and the rubber matrix was determined for the middle part (dynamic adhesion strength) and for the end parts (static adhesion strength) using an Instron 4465 Universal Tester with a $5 \mathrm{kN}$ force head at crosshead speeds of $10 \mathrm{~mm} \cdot \mathrm{min}^{-1}$.

\section{Characterisation of PET morphology}

The morphology of the PET cords was investigated by SEM and atomic force microscopy (AFM). SEM was performed in a Tesla BS 300 instrument using a voltage of $15 \mathrm{kV}$. Before the measurement, the samples were coated with a gold and platinum blend in a Balzer SCD 050. AFM studies were performed on ultramicrotomed samples using a Multimode Nanoscope IIIa model in the phase mode. Force modulation SPM tips were used. The tip was mounted on a $225 \mathrm{~mm}$ (length), $3 \mathrm{~mm}$ (thickness) single beam cantilever, and vibrated with resonant frequencies of $400 \mathrm{kHz}$ and a force constant of $2.8 \mathrm{~N} \cdot \mathrm{m}^{-1}$. The tip height was in the range of $10-15 \mathrm{~mm}$ with a sharp edge of $10 \mathrm{~nm}$.

\section{Measurement of mechanical properties of PET cords}

The mechanical properties were measured using an Instron 4465 Universal Tester (ASTM D 638M) with a $5 \mathrm{kN}$ force head at crosshead speeds of $10 \mathrm{~mm} \cdot \mathrm{min}^{-1}$.

\section{Measurement of gas products during the plasma polymerization}

Spectrum of gas products arise by decomposition of butadiene in spark corona discharge at atmospheric pressure were measured with UV-VIS spectrometer JobinYvon HR640 with grid 1200 scratch per millimeter.

\section{Chemical analysis}

The XPS analysis of the samples was carried out on a Multiprobe MXPS from OMICRON. A five channel, $180^{\circ}$ hemispherical analyser was used. The detection area was $0.5 \mathrm{~cm}^{2}$, and an emission angle of $45^{\circ}$ was used to observe chemical changes of the polymeric substrate to a depth of 3-6 nm.

\section{Results and Discussion}

\section{Plasma treatment}

Setup of the plasma reactor made it possible to adjust the speed at which the cords move through the reactor, the influence of treatment time on the static and dynamic adhesion strength of the cords was investigated (Table 1). It is evident that the adhesion strength is best when the speed of the fibres was $13.8 \mathrm{~m} \cdot \mathrm{min}^{-1}$ and the distance at $1.5 \mathrm{~cm}$ between the electrodes. Under these conditions the contact of the plasma with the PET cords was only $0.06 \mathrm{~s}$. From Table 1 is also evident that at all the cords speeds through the reactor, the static adhesion strength values are higher than the dynamic adhesion strength values. However, after plasma treatment the gap between the static and dynamic values is much smaller (the difference between the static and dynamic adhesion strength for the untreated PET fibres is $\sim 50 \mathrm{~N}$, while for the treated fibres it is only $\sim 20 \mathrm{~N}$ ). This further confirms that the plasma has a substantial influence on the adhesion strength.

The experimental results obtained from treatment by coplanar dielectric surface barrier discharge in nitrogene plasma are shown in Figure 3 and in ambient air plasma in Figure 4.

As it is shown in figures, the maximal adhesion of PET cords activated in CDSBD discharge in ambient air at atmospheric pressure. In the case was obtained $94 \%$ of static adhesion and $96 \%$ of dynamic adhesion in comparison with adhesion when RFL was used. From Figure 3 is evident that the maximal adhesion of PET cords activated in nitrogen was $116 \%$ in the case of static

Table 1. The influence of speed of PET cord movement in the atmospheric reactor on the static and dynamic adhesion strength (nitrogen plasma).

\begin{tabular}{ccccc}
\hline $\begin{array}{c}\text { Speed of cord movement } \\
{\left[\mathrm{m} \cdot \mathrm{min}^{-1}\right]}\end{array}$ & $\begin{array}{c}\text { Static adhesion strength } \\
{[\mathrm{N}]}\end{array}$ & Deviation & $\begin{array}{c}\text { Dynamic adhesion strength } \\
{[\mathrm{N}]}\end{array}$ & Deviation \\
\hline 0.0 (Untreated cord) & 53.0 & 3.1 & 7.3 & 1.2 \\
2.0 & 109.1 & 5.5 & 90.6 & 5.3 \\
13.8 & 133.9 & 4.8 & 125.0 & 6.3 \\
31.0 & 118.0 & 4.8 & 102.0 & 4.1 \\
48.2 & 115.0 & 6.8 & 108.9 & 5.8 \\
60.0 & 114.9 & 3.3 & 101.2 & 3.8 \\
\hline
\end{tabular}




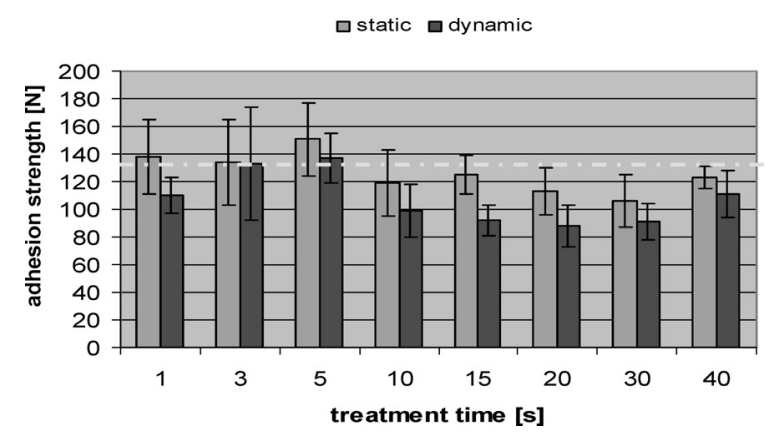

Figure 3. Dependence of adhesion strength of PET cords to rubber on activation time in nitrogen plasma generated by DCSBD.

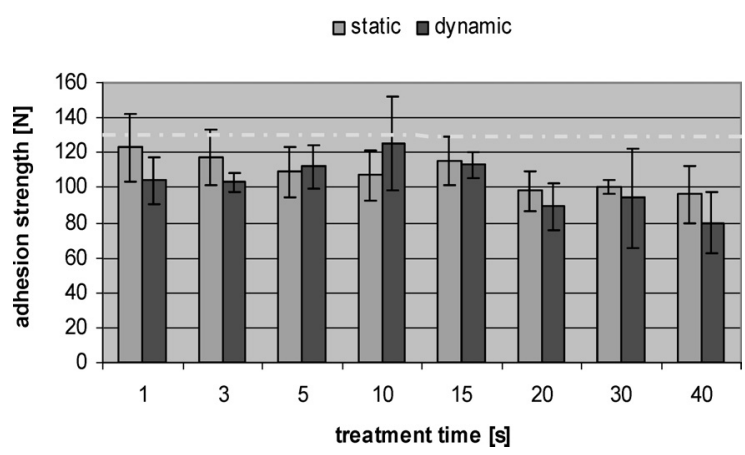

Figure 4. Dependence of adhesion strength of PET cords to rubber on activation time in ambient air plasma generated by DCSBD.

adhesion and $105 \%$ in the case of dynamic adhesion in comparison with adhesion when RFL was used. These changes were already obtained with activation for several seconds. As Krump et al. ${ }^{6}$ mentioned before, under sufficient conditions, the relatively short contact between the PET fibre and plasma is sufficient. Higher exposure of treatment caused decrease of adhesion strength.

It is known that plasma treatment changes the polymer surface, especially the morphology. It depends on the nature of the gas, time of treatment, as well as the performance of the reactor. When treated, the roughness of the polymeric material increased, as observed from scanning electron or atomic force microscopy. When the roughness increases, the polymer surface becomes larger, giving rise to a larger contact area and better adhesion towards other materials. Our PET fibres were treated in an atmospheric plasma reactor, using argon and nitrogen as plasma gases. The highest roughness was observed when the speed of the fibre inside the reactor was $13.8 \mathrm{~m} \cdot \mathrm{min}^{-1}$. From Figure 5 it is evident that the argon plasma has a slight effect on the morphology .

The highest roughness was observed when nitrogen was used as plasma gas, which confirmed that nitrogen is a more active gas than the inert argon. Roughness is one of

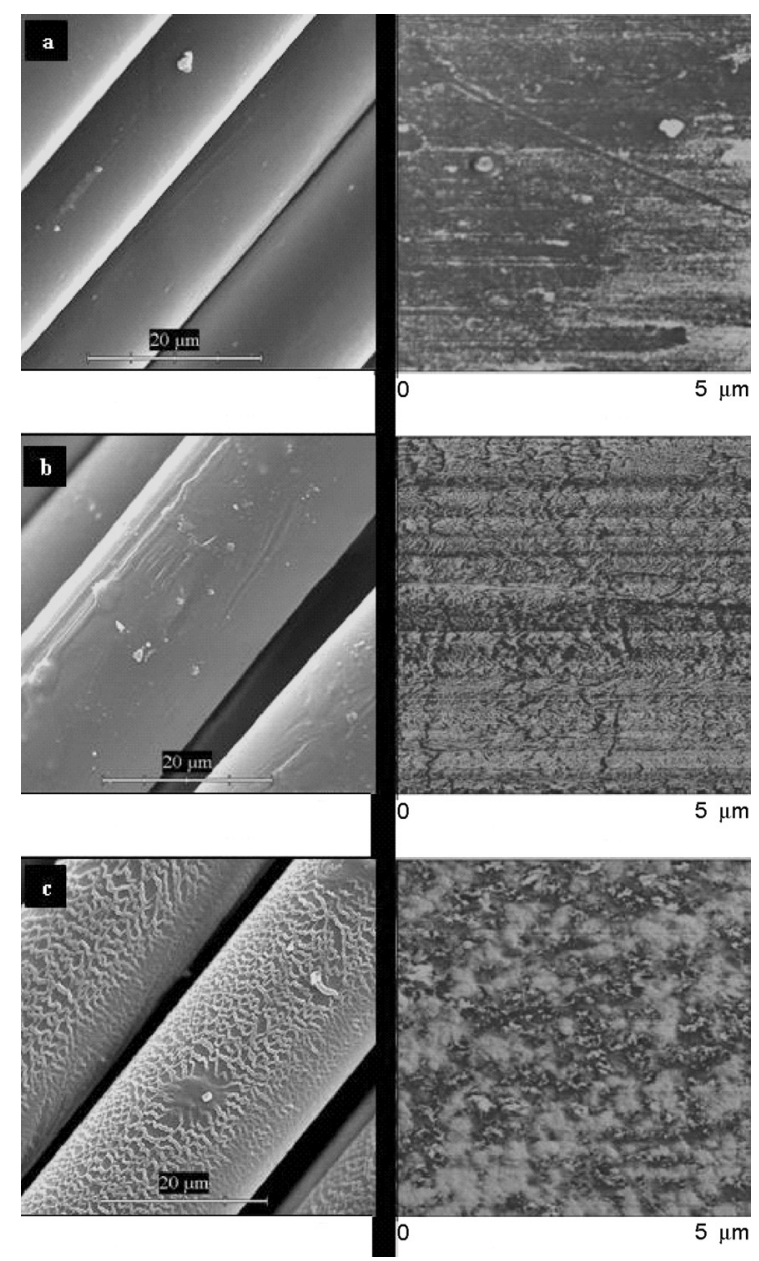

Figure 5. Scanning electron micrographs (SEM, left column) and atomic force micrographs (AFM, right column) of (a) untreated PET fibre, (b) PET fibre after activation in argon plasma, (c) PET fibre after activation in nitrogen plasma.

the factors that have a substantial influence on the adhesion properties between PET fibres and a polymer matrix.

When treated, there is not only a change in the surface properties, but a very thin, active layer is also formed below the polymer surface. The thickness of this layer, the modified depth, depends on the chemical nature of the polymeric materials (linear or branch chains, functional groups, crystallinity), as well as on the setup of the reactor, treatment time, and type of gas used. The thickness of the modified layer is several hundreds of $\AA$, and it is possible that the plasma treatment has an influence on the mechanical properties (strength and elongation at break, modulus) of the PET fibres. This influence can be seen during the treatment of very thin substrates, but the plasma treatment of a material like PET fibre (thickness of about $0.5 \mathrm{~mm}$ ) should have no influence on the mechanical 
Table 2. Comparison of the mechanical properties of the PET fibres (untreated, after $\mathrm{N}_{2}$ plasma treatment, with RFL system).

\begin{tabular}{lcccc}
\hline Type of treatment & $\begin{array}{c}\text { Speed of the PET fibers } \\
\text { in the reactor } \\
{\left[\mathrm{m} \cdot \mathrm{min}^{-1}\right]}\end{array}$ & $\begin{array}{c}\text { Strength at break } \\
{[\mathrm{N}]}\end{array}$ & $\begin{array}{c}\text { Elongation at break } \\
{[\%]}\end{array}$ & $\begin{array}{c}\text { Elongation } \\
\text { under weight } 45 \mathrm{~N} \\
{[\%]}\end{array}$ \\
\hline Untreated PET fibre & - & $176.8 \pm 2.2$ & $15.3 \pm 0.5$ & $\begin{array}{c}\text { Elongation } \\
\text { under weight 90 N } \\
{[\%]}\end{array}$ \\
PET fibre $+\mathrm{N}_{2}$ plasma & 2 & $177.4 \pm 1.8$ & $16.3 \pm 0.4$ & $3.6 \pm 0.3$ \\
PET fibre $+\mathrm{N}_{2}$ plasma & 4 & $177.0 \pm 1.2$ & $14.4 \pm 0.7$ & $4.2 \pm 0.2$ \\
PET fibre $+\mathrm{N}_{2}$ plasma & 10 & $175.9 \pm 2.0$ & $15.4 \pm 1.0$ & $3.5 \pm 0.3$ \\
PET fibre $+\mathrm{N}_{2}$ plasma & 13.8 & $176.3 \pm 2.1$ & $15.5 \pm 0.7$ & $3.8 \pm 0.2$ \\
PET fibre $+\mathrm{N}_{2}$ plasma & 60 & $176.4 \pm 0.6$ & $16.2 \pm 0.5$ & $3.7 \pm 0.2$ \\
PET fibre + RFL system & - & $179.0 \pm 3.3$ & $17.3 \pm 1.2$ & $3.9 \pm 0.3$ \\
\hline
\end{tabular}

properties. The results (Table 2) confirm that atmospheric plasma treatment does not have any influence on the mechanical properties of the PET fibres.

\section{Plasma polymerization}

According to Yasuda and $\mathrm{Hsu}^{7)}$ which introduced a new concept of plasma polymerization is the plasma responsible for the fragmentation of molecules. In plasma, monomer molecules gain high energy from electrons, ions and radicals which are fragmented into activated small fragments, in some cases into atoms. These activated fragments are recombined, sometimes accompanying rearrangement, and the molecules grow to high-molecularweight polymer in a gas phase or at the surface of a substrate. If energy supplied from high voltage source is sufficient for ionization of nitrogen, we will expect that the molecules of butadiene gas have been also fragmented in plasma. This assumption confirmed by measured spectra of gas products arise by decomposition of butadiene in pulse discharge at atmospheric pressure (Figure 6), where we detected the strips of ionized nitrogen, oxygen, hydrogen and also the new formed functional groups as $\mathrm{CN}$, what confirmed the Yasudas atomic plasma polymerization theory. The new polymer layer was also detected by SEM (Figure 7).

In the experiment we studied the influence of: power of reactor and speed of cord movement trough reactor to adhesion to rubber (Figure 8).

To assess the effect of the plasma treatment on cord surface properties, the measured values of adhesion were compared with adhesion of RFL treated cords (chemical modification of PET cords usually used in tire industry). It is evident that adhesion of plasma modified cords is comparable with adhesion achieve with RFL treated cords which presented the dashed line. In the specific setup of experiment even we can achieve higher adhesion compared with RFL treatment.

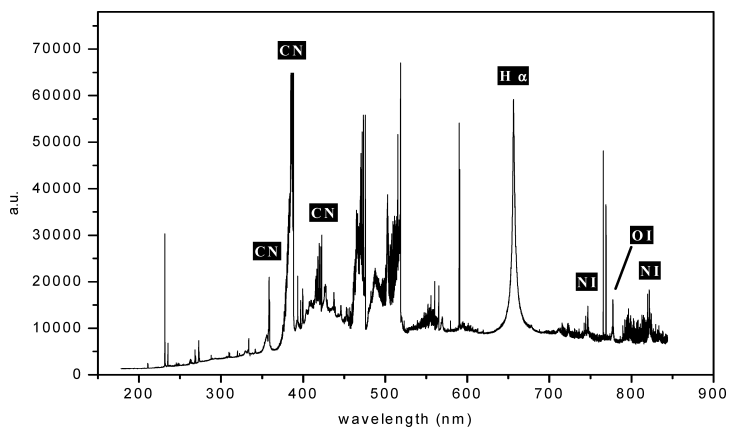

Figure 6. Spectrum of gas product arise by decomposition of butadiene in pulse corona discharge at atmospheric pressure.
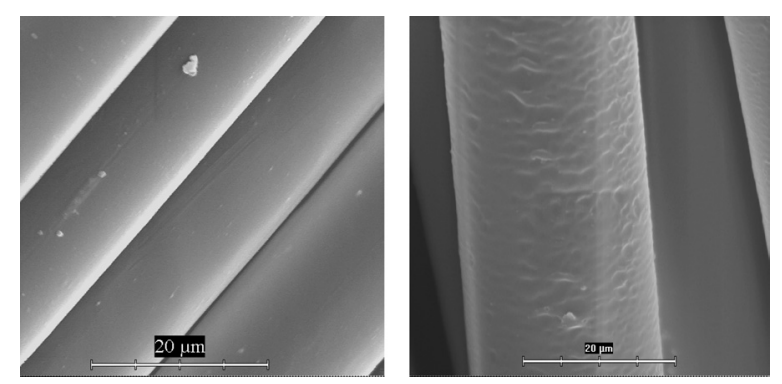

Figure 7. SEM of untreated PET cord (left) and PET cord after plasma polymerization (right).
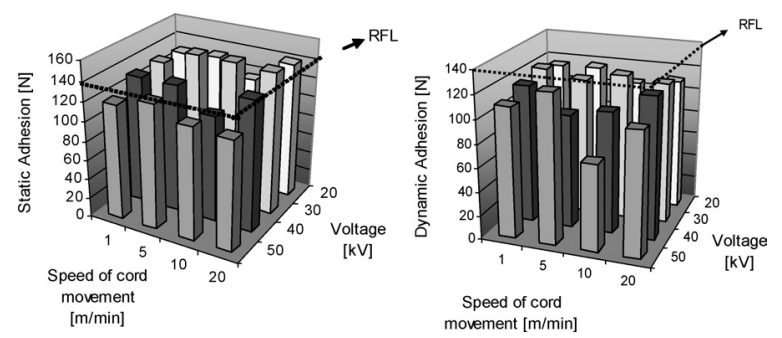

Figure 8. Static and dynamic adhesion strength of modified PET cords after plasma polymerization of butadiene in mixture with nitrogene (butadiene flow rate $0.1 \mathrm{dm}^{3} \cdot \mathrm{min}^{-1}$ and nitrogen flow rate $\left.3 \mathrm{dm}^{3} \cdot \min ^{-1}\right)$. 


\section{Conclusions}

The main aim of this work was to receive a more detail information regarding to nitrogen and ambient air plasma treatment on the PET cords in two type of atmospheric plasma reactors. There is evident that the plasma treatment has substantial effect on the increase of adhesion strength towards rubber blend.

Negligible changes in mechanical properties were observed but these changes do not have any affect for its industrial application. Also changes in morphology of modified fibres can increase the adhesion strength to a rubber.

In the last we observed that the plasma polymerization in the butadiene-nitrogen gas mixture creat of new polymer layer on the cord surface. The obtained results for the surface modification of PET cords by butadiene plasma polymerization with pulse surface positive corona discharge at atmospheric pressure shows, that the modified cords exhibit dynamic and static adhesion comparable with the standard industrial chemical treatment based on RFL. Negligible changes in mechanical properties were observed however these changes have not affect for its industrial application.

Finally utilisation of plasma can simplified the surface modification of polyester cords and increased the adhesion to rubber, out of consideration to environment.

\section{References}

1) Janssen H.: Kautschuk Gummi Kunststoffe, 48, 622-624 (1995).

2) Ooij W. J. van, Luo S., Datta S.: Plasmas and Polymers, 4(1), 33-55 (1999).

3) Štefecka M., et al.: J. Mater. Sci. Lett., 18, 2007 (1999).

4) Štefecka M., et al.: J. Mater. Sci. Lett., 19, 1869 (2000).

5) Krump H., et al.: Elastomer, 37(3), 192-194 (2002).

6) Krump H., et al.: Appl. Surface Sci., 240, 268-274 (2005).

7) Yasuda H., Hsu T.: J. Polym. Sci. Chem. Ed., 15, 81 (1977).

The paper as-received has been presented at IRC 2005 Yokohama held on Oct. 24-28, 2005. 